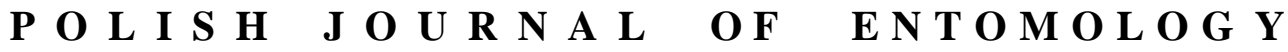

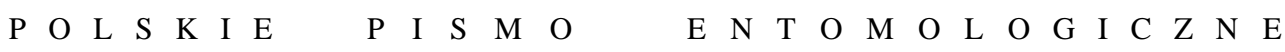

VOL. 83: $141-170$

Lublin

30 June 2014

DOI: $10.2478 /$ pjen-2014-0011

\section{Contributions to the knowledge the scarab beetles of Golestan province, Northern Iran with checklist of Iranian Scarabaeoidea (Coleoptera)}

\author{
MAREK BUNALSKI ${ }^{1}$, NAJMEH SAMIN ${ }^{2}$, HASSAN GHAHARI ${ }^{3}$, TREVOR J. HAWKESWOOD ${ }^{4}$ \\ ${ }^{1}$ Department of Entomology and Environmental Protection, University of Life Sciences, \\ Dąbrowskiego 159, 60-594 Poznań, Poland, email: bunalski@up.poznan.pl \\ ${ }^{2}$ Young Researchers Club and Elites, Science and Research Branch, Islamic Azad \\ University, Tehran, Iran, e-mail: n_samin63@yahoo.com \\ ${ }^{3}$ Department of Plant Protection, Shahre Rey Branch, Islamic Azad University, Tehran, \\ Iran, email: hghahari@yahoo.com \\ ${ }^{4}$ PO Box 842, Richmond, New South Wales, Australia, \\ email: drtjhawkeswood@calodema.com
}

\begin{abstract}
This paper deals with the fauna of Scarabaeoidea (Coleoptera) in Golestan province, Northern Iran. In total 19 species from 16 genera (Adoretus, Aphodius, Amphimallon, Cetonischema, Copris, Eulasia, Euonthophagus, Glaphyrus, Netocia, Onthophagus, Oxythyrea, Pentodon, Valgus, Polyphylla, Stalagmosoma, Trichius) were collected and recorded from this region. A checklist of Iranian Scarabaeoidea is provided.
\end{abstract}

KEY WORDS: Coleoptera, Scarabaeoidea, Golestan province, Iran, checklist.

\section{INTRODUCTION}

The superfamily Scarabaeoidea comprises worldwide more than 35000 species (RATCLIFFE \& PAULSEN 2008). The family Scarabaeidae as presently defined consists of over 30000 species of beetles worldwide (RATCLIFFE et al. 2002). Scarab beetles are one of the most important insect groups, and they are a conspicuous component of the beetle fauna in almost all ecosystems (WOODRUFF 1973). Scarabs are stout-bodied beetles, many with bright metallic colors, measuring between 5-60 $\mathrm{mm}$. They have distinctive, clubbed 
antennae composed of plates called lamellae that can be compressed into a ball or fanned out like leaves to sense odors (NiKOlayev 1987, RATCLifFE et al. 2002). The fauna of Iranian Scarabaeoidea was poorly studied, with a total of 150 species recorded by Modarres Awal (1997) and about 20 species in other papers until that time (PETrovitz 1954, 1958, 1980; BARAUD 1968, BORTESI \& ZUNINO 1974, ZAIRI 1976, KABAKOV 1982, NIKODÝM \& KRÁL 1998). Since 1997, nearly 40 species have been recorded from different authors (BARARI et al. 1998a, 1998b, BARARI 2001, KeITH \& AHRENS 2002, ModARRES AwAl 2006, ZiAni \& GUdENZI 2000, 2006, 2007, 2009, ZIANI 2006, 2011, MONTREUIL \& Serri 2007, Keith \& Uliana 2008, Mowlavi et al. 2008, Moradi GharaKhloo \& ZiANi 2009, 2010, Ziani \& Moradi GHARAKHLOO 2010, GHAHARI et al. 2011, YARMAND et al. 2012). In addition, several species are listed in the Catalogue of Palaearctic Coleoptera (LÖBL \& SMETANA 2006).

Golestan province is located in the north of Iran and south of the Caspian Sea (Fig.). Geographically, Golestan is divided into two sections, the plains and the mountains of the Alborz range. In the eastern Alborz section, the mountains have a north-easterly aspect and gradually decreases in height. The highest point of the province is Shavar $-3945 \mathrm{~m}$ above sea level. The climate of Golestan is temperate for most of the year. The aim of this paper is faunistic survey on Scarabaeoidea in some regions of Golestan province (northern Iran).

\section{Acknowledgements}

The authors are grateful to Dr. M. UliANA and Dr. S. ZIANI of Italy for their invaluable assistance during this research, and Dr. H. SAKENIN for the loan of many specimens. The research was supported by Islamic Azad University (Young Researchers Club and Elites, and Shahre Rey Branch) and Poznań University of Life Sciences.

\section{MATERIALS AND METHODS}

The materials were collected from some regions of Golestan province (northern Iran) during 2005-2010. The collecting methods included sweeping net, light traps and knock down. All the specimens collected were put in alcohol (ethanol 75\%) and identified by the authors. Information concerning date of collection, locality, altitude and number of specimens (in brackets) are given. The classification and nomenclature of scarab beetles suggested by LÖBL \& SMETANA (2006) have been followed.

All specimens have been deposited in the authors' collections. 


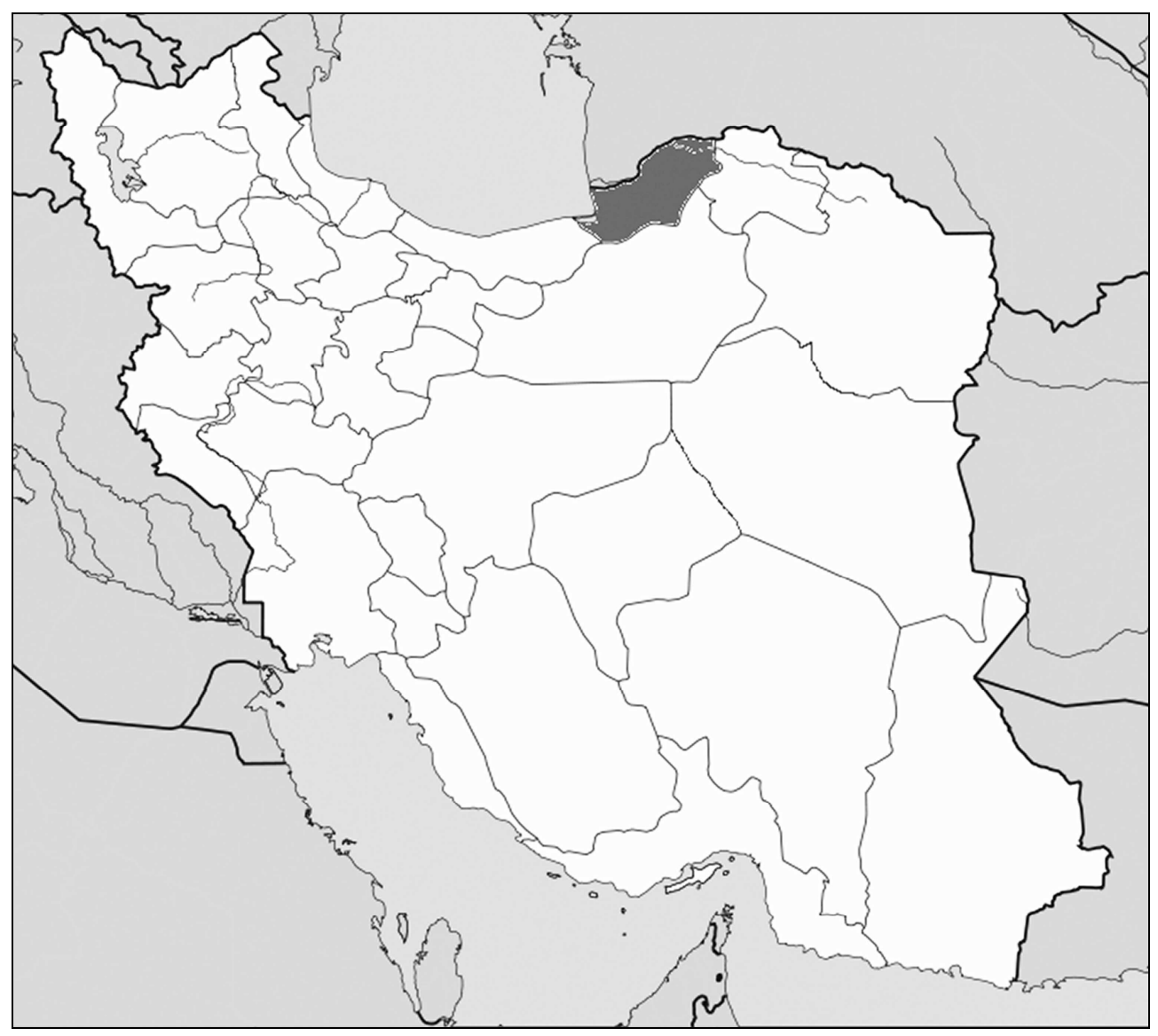

Fig. Map of Iran showing the location of Golestan Province. (source: Wikipedia).

\section{RESULTS AND DISCUSSION}

Among the collected specimens of Scarabaeoidea from Golestan province, totally 19 species from 16 genera were identified. All species are new for Golestan province and one new for Iranian fauna. Since Golestan province boasts a diverse flora and various geographical regions (HASSAN ZADEH et al. 1993), a diverse fauna of Scarabaeoidea is expected for this region and requires further study. Although one species of this research is new country record, such faunistic works are valuable for advancing the knowledge of specialists on the Iranian fauna. Determining the main host plants of Scarabaeoidea and also the most important natural enemies of these insects can be valuable research topics. The list of species (19 species from 16 genera) is given below. 


\section{Family: Glaphyridae MACLEAY, 1819}

Eulasia (Eulasia) azarbaijanica (PETROVITZ, 1980)

Distribution: Iran.

Material: Golestan National Park, 32 m, (1), July 2005.

Remarks: new to Golestan province.

Glaphyrus oxypterus (PALLAS, 1771)

Distribution: Southern Russia, Kazakhstan.

Material: Gorgan, near Nahar-Khoran, 450 m, (2), 28 May 2010.

Remarks: new to Iranian fauna.

Family: Scarabaeidae LATREILLE, 1802

Subfamily: Aphodiinae LEACH, 1815

Tribe: Aphodiini LEACH, 1815

Aphodius (Biralus) menetriesi MÉNÉTRIES, 1849

Distribution: Central and Southern Russia, Central Asia, Iran, Turkey.

Material: 45 km E of Minoo-Dasht, 960 m, (1), 11 June 2010.

Remarks: new to Golestan province.

\section{Subfamily: Scarabaeinae LATREILLE, 1802}

Tribe: Coprini LEACH, 1815

Copris lunaris (LINNAEUS, 1758)

Distribution: Europe, Turkey, Iran, Central Asia, China.

Material: Bandar-Gaz, 6 m, (2), April 2010; Gonbad, 11 m, (1), July 2010.

Remarks: new to Golestan province.

\section{Tribe: Onthophagini BURMEISTER, 1846}

Euonthophagus gibbosus (SCRIBA, 1790)

Distribution: Southern and South-Eastern Europe, Turkey, Near East, Iran, Afghanistan,

Central Asia, China.

Material: Galikesh, 132 m, (3), October 2010.

Remarks: new to Golestan province.

Onthophagus (Palaeonthophagus) ruficapillus BRULLÉ, 1832

Distribution: Southern and Central Europe, Turkey, Near East, Iran, Iraq.

Material: Minoo-Dasht, 175 m, (1), September 2006; Ali-Abad, 130 m, (1), May 2010.

Remarks: new to Golestan province. 
Onthophagus (Palaeonthophagus) vacca (LINNAEUS, 1767)

Distribution: Europe, Turkey, Near East, Iran, Turkmenistan.

Material: Golestan National Park, Sulgard, 1847 m, (1), September 2010.

Remarks: new to Golestan province.

\section{Subfamily: Melolonthinae SAMOUELLE, 1819}

Tribe: Melolonthini SAMOUELLE, 1819

Polyphylla (Polyphylla) olivieri LAPORTE, 1840

Distribution: Graecia, Turkey, Near East, Iran, Transcaucasia.

Material: Minoo-Dasht, 178 m, (3), September 2006; Golestan Natural Park, S of Qalan, 1851 m, (1), August 2007; Kalaleh, 135 m, (2), August 2009; Gomishan, -4 m, (1),

April 2010; Golestan National Park, N of Sulgard, 1944 m, (2), October 2010.

Remarks: new to Golestan province.

Polyphylla (Xerasiobia) adspersa MoTSCHULSKY, 1853

Distribution: Transcaucasia, Turkey, Iran, Afghanistan, Central Asia.

Material: Bandar-Torkaman, 18 m, (2), August 2010; Galikesh, 117 m, (1), October 2010.

Remarks: new to Golestan province.

\section{Tribe: Rhizotrogini BURMEISTER, 1855}

Amphimallon leuthneri REITER, 1902

Distribution: Turkey, Syria, Iran.

Material: Golestan National Park, N of Armodlu, (3), August 2007.

Remarks: new to Golestan province.

\section{Subfamily: Rutelinae MACLEAY, 1819 \\ Tribe: Adoretini BURMEISTER, 1844}

Adoretus nigrifrons (STEVEN, 1809)

Distribution: Southern Russia, Transcaucasia, Iran, Afghanistan, Central Asia, Western China.

Material: 10 km S of Azad-Shahr, 284 m, (1), 11 June 2010; Bandar-Torkaman, 22 m, (1), August 2010.

Remarks: new to Golestan province.

\section{Subfamily: Dynastinae MACLEAY, 1819 \\ Tribe: Pentodontini Mulsant, 1842}

Pentodon algerinus bispinifrons REITTER, 1894

Distribution: Azerbaijan, Iran, Afghanistan, Kazakhstan, Central Asia, Western China. 
Material: Gorgan, 141 m, (1), June 2007; Ramyan, 220 m, (1), July 2010.

Remarks: new to Golestan province.

Pentodon bidens sulcifrons KUSTER, 1848

Distribution: Bulgaria, Graecia, Turkey, Near East, Iran, Iraq, Transcaucasia.

Material: Golestan National Park, 32 m, (1), July 2005.

Remarks: new to Golestan province.

\section{Subfamily: Cetoniinae LEACH, 1815}

Tribe: Cetoniini LEACH, 1815

Oxythyrea cinctella cinctella (SCHAUM, 1841)

Distribution: South-Eastern Europe, Transcaucasia, Turkey, Near East, Middle East,

Afghanistan, Kazakhstan, Central Asia, Western China.

Material: Golestan National Park, 32 m, (6), July 2005.

Remarks: new to Golestan province.

Protaetia (Cetonischema) speciosa (ADAMs, 1817)

Distribution: South-Eastern Europe, Transcaucasia, Iran, Turkey, Near East.

Material: Maraveh-Tappeh, 218 m, (2), July 2010.

Remarks: new to Golestan province.

Protaetia (Netocia) ungarica ignisternum REITTER, 1891

Distribution: Turkey, Iran, Azerbaijan, Iraq, Turkmenistan.

Material: Gorgan, 64 m, (1), June 2010.

Remarks: new to Golestan province.

Stalagmosoma albellum (PALLAS, 1781)

Distribution: Transcaucasia, Turkey, Near East, Iran, Iraq, Afghanistan, Central Asia.

Material: Golestan National Park, S of Qalan, (1), August 2007.

Remarks: new to Golestan province.

\section{Tribe: Trichini}

Trichius abdominalis MÉNÉTRIES, 1832

Distribution: Azerbaijan, Iran.

Material: Golestan National Park, 32 m, (2), July 2005.

Remarks: new for Golestan province. 


\section{Tribe: Valgini MulSANT, 1842}

Valgus hemipterus (LINNAEUS, 1758)

Distribution: Europe, Southern Russia, Iran, Central Asia, North Africa.

Material: Golestan National Park, N of Yaghtiklan, (2), August 2007.

Remarks: new to Golestan province.

Owing to a high habitat diversity, Iranian wildlife is very rich and diversified. This trend is also applicable to Scarabaeoidea, whose checklist includes 509 species and subspecies belonging to 107 genera (see checklist). Unfortunately, the number of recorded species is not on a par with the knowledge on their ranges and habitat relationships. Iran has 31 administrative provinces, but faunistic information about scarab beetles has been gathered only from a dozen of them, and a preliminary inventory has been made for just a few areas. This underlines the necessity to intensify research, particularly faunistic and ecological studies.

\section{CHECKLIST OF IRANIAN SCARABAEOIDEA}

\section{Family: Lucanidae LATREILLE, 1804}

Subfamily: Aesalinae MACLEAY, 1819

Genus: Aesalus FABRICIUS, 1801

* ulanowskii GANGLBAUER, 1886

Subfamily: Synodesinae MACLEAY, 1819

Genus: Sinodendron HeLlWIG, 1792

* persicum REITTER, 1902

Subfamily: Lucaninae LATREILLE, 1804

Genus: Lucanus SCOPOLI, 1763

* cervus cervus (LINNAEUS, 1758)

* ibericus Motschulsky, 1845

* orientalis KRAATZ, 1860

Genus: Platycerus GEOFFROY, 1762

* caraboides caraboides (LINNAEUS, 1758)

Genus: Pseudolucanus HoPE et WestwOOD, 1845

* busignyi PlANET, 1897 
Subfamily: Dorcinae PARRY, 1864

Genus: Dorcus MACLEAY, 1819

* parallelipipedus (LINNAEUS, 1758)

* peyroni ReICHE et SAULCY, 1856

* prochazkai SCHENK, 2003

* vavrai NONFRIED, 1905

\section{Family: Trogidae MACLEAY, 1819}

Genus: Afromorgus SCHOLTZ, 1986

* procerus (HAROLD, 1872)

Genus: Trox FABRICIUS, 1775

* eversmanni KRYNICKY, 1832

* hispidus (PONTOPPIDAN, 1763)

* iranicus Petrovitz, 1980

* klapperichi PITTINO, 1983

* quadrimaculatus BALLION, 1871

\section{Family: Glaresidae KoLBE, 1905}

Genus: Glaresis ERICHSON, 1848

* beckeri SOLSKY, 1870

* freyi PETROVITZ, 1968

* porrecta (SEMENOV et MEDVEDEDV, 1932)

* zarudniana (SEMENOV et MEDVEDEDV, 1932)

\section{Family: Bolboceratidae}

Tribe: Australobolbini NiKOLAJEV, 1996

Genus: Bolboceras KIRBY, 1819

* davatchii BARAUD, 1973

Tribe: Eubolbitini NIKOLAJEV, 1970

Genus: Eubolbitus REITTER, 1892

* radoszkowskii (SOLSKY, 1876)

* zarudnyi SEMENOV et MEDVEDEDV, 1929

\section{Family: Geotrupidae LATREILLE, 1802}

Subfamily: Geotrupinae LATREILLE, 1802

Tribe: Geotrupini LATREILLE, 1802

Genus: Geotrupes LATREILLE, 1797

* mutator (MARSHAM, 1802) 
* olgae (OLSOUFIEFF, 1918)

* spiniger (MARSHAM, 1802)

* stercorarius (LINNAEUS, 1785)

Genus: Trypocopris MOTSCHULSKY, 1860

* fausti (REITTER, 1890)

Subfamily: Lethrinae MuLSANT et REY, 1871

Genus: Lethrus SCOPOLI, 1777

Subgenus: Lethrus SCOPOLI, 1777

* acutangulus BALLION, 1871

Subgenus: Scelolethrus SEMENOV, 1892

* chorassanicus SEMENOV et MEDVEDEDV, 1935

* mithras REITTER, 1904

Family: Ochodaeidae MulSANT et REY, 1871

Subfamily: Ochodaeinae MULSANT et REY, 1871

Genus: Ochodaeus DEJEAN, 1821

* cornifrons SOLSKY, 1876

* solskyi SEMENOV, 1891

\section{Family: Hybosoridae ERICHSON, 1847}

Subfamily: Dynamopodinae ARROw, 1911

Genus: Orubesa REITTER, 1895

* ata (SEMENOV et MEDVEDEV, 1929)

* luctator (SEMENOV et MEDVEDEV, 1929)

* perforata $\mathrm{REITTER}, 1895$

Subfamily: Hybosorinae ERICHSON, 1847

Genus: Hybosorus MACLEAY, 1819

* illigeri REICHE, 1853

\section{Family: Glaphyridae MACLEAY, 1819}

Genus: Eulasia TRUQUI, 1848

Subgenus: Eulasia TRUQUI, 1848

* aurantiaca (REITTER, 1890)

* azarbaijanica (PETROVITZ, 1980)

* bodemeyeri (PETROVITZ, 1965)

* bombyliformis bombyliformis (PALLAS, 1781) 
* chrysopyga (FALDERMANN, 1835)

* diadema (REITTER, 1890)

* naviauxi (BARAUD, 1971)

* nitidinatis BARAUd, 1990

* persidis BaRAUd, 1990

* praeusta (CHAMPENOIS, 1896)

* zaitzevi (BogatcheV, 1947)

Subgenus: Redeulasia BARAUD, 1990

* bocquilloni (ZAITZEV, 1923)

* carinata BARAUD, 1990

* chalybaea chalybaea (FALDERMANN, 1835)

* genei TRUQUI, 1848

* papaveris (STURM, 1843)

* pulchra kurdistana (Petrovitz, 1968)

* pulchra pulchra (REITTER, 1890)

* speciosa (CHAMPENOIS, 1900)

* straussi (GANGLBAUER, 1905)

Subgenus: Solskiola SEMENOV, 1903

* analis (SOLSKY, 1876)

Subgenus: Trichopleurus MOTSCHULSKY, 1860

* vittata lineata (FALDERMANN, 1835)

* vittata persica (Petrovitz, 1963)

Genus: Glaphyrus LATREILLE, 1807

Subgenus: Glaphyrus LATREILLE, 1807

* festivus MÉNÉTRIES, 1836

* laufferi ReITTER, 1903

* luristanus ReITter, 1903

* micans micans FALDERMANN, 1835

* muticus ReITter, 1903

* onopordi ReITTER, 1903

* oxypterus (PALLAS, 1771)

* sequensi ReITTER, 1903

* superbus straussi ReITTER, 1903

* superbus superbus CHAMPENOIS, 1898

* varians varians MENETRIES, 1836

Subgenus: Hemiglaphyrus CHAMPENOIS, 1903

* caucasicus KRAATZ, 1882 


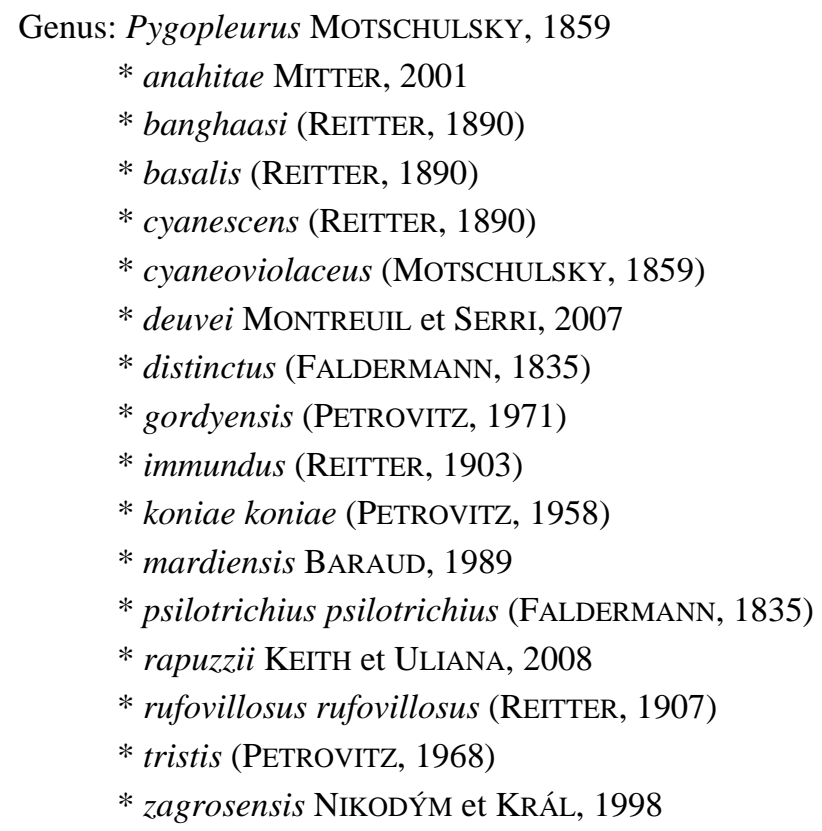

\section{Family: Scarabaeidae LATREILLE, 1802}

Subfamily: Eremazinae IABLOKOFF-KHNZORIAN, 1977

Tribe: Eremazini IABLOKOFF-KHNZORIAN, 1977

Genus: Eremazus MULSANT, 1851

* cribratus SEMENOV, 1893

* unistriatus MULSANT, 1851

Subfamily: Aphodiinae LEACH, 1815

Tribe: Aphodiini LEACH, 1815

Genus: Aphodius ILLIGER, 1798

Subgenus: Acanthobodilus G. DELlaCASA, 1983

* immundus CREUTZER, 1799

Subgenus: Acrossus MulsAnT, 1842

* depressus (KugELANN, 1792)

* gagatinus MÉNÉTRIES, 1832

Subgenus: Alocoderus A. SCHMIDT, 1913

* hydrochaeris (FABRICIUS, 1798)

* strigimargo REITTER, 1892

* turbatus BAUDI, 1870 
Subgenus: Amidorus MulsanT et ReY, 1870

* obscurus obscurus (FABRICIUS, 1792)

Subgenus: Ammoecius MuLSANT, 1842

* meurguesae CLEMENT, 1975

Subgenus: Aphodaulacus W. KosHANTSCHIKOV, 1911

* turkestanicus HEYDEN, 1881

Subgenus: Aphodius ILLIGER, 1798

* fimetarius (LINNAEUS, 1758)

Subgenus: Biralus MulsanT et REY, 1869

* equinus FALDERMANN, 1835

* menetriesi MÉNÉTRIES, 1849

Subgenus: Bodilus Mulsant et REY, 1870

* asiricus PITTINO, 1984

* gregarius HAROLD, 1871

* ictericus ghardimaouensis BALTHASAR, 1929

* kermanschahensis Petrovitz, 1959

* lugens CREUTZER, 1799

* punctipennis ERICHSON, 1848

* wollastoni iranicus BALTHASAR, 1946

* zarudnyi FROLOv, 2001

Subgenus: Calamosternus MOTSCHULSKY, 1859

* granarius (LINNAEUS, 1758)

* hyxos Petrovitz, 1962

* machulkai Balthasar, 1935

* trucidatus HAROLD, 1863

* unicolor (OLIVIER, 1789)

* vexator BALTHASAR, 1933

Subgenus: Chilothorax MOTSCHULSKY, 1860

* clathratus REITTER, 1892

* discedens SCHMIDT, 1907

* hieroglyphicus KLUG, 1845

* melanostictus W.L. SCHMIDT, 1840

Subgenus: Colobopterus MULSANT, 1842

* erraticus (LinNAEUS, 1758)

Subgenus: Coprimorphus MULSANT, 1842

* scrutator (HERBST, 1789) 
Subgenus: Erytus MuLSANT et REY, 1870

* aequalis A. SCHMIDT, 1907

* bucharicus PETROVITZ, 1961

* cognatus FAIRMAIRE, 1860

* hormonzensis PETROVITZ, 1980

* lindemannae BALTHASAR, 1960

* persicus PETROVITZ, 1961

* pruinosus REITTER, 1892

Subgenus: Esymus MULSANT et REY, 1870

* merdarius (FABRICIUS, 1775)

* pusillus pusillus (HERBST, 1789)

* suturinigra A. SCHMIDT, 1916

Subgenus: Eudolus MulsANT et REY, 1870

* quadriguttatus (HERBST, 1783)

Subgenus: Euorodalus G. DEllaCASA, 1983

* paracoenosus BALHASAR et HRUBANT, 1960

Subgenus: Labarrus MulSANT et REY, 1870

* digitatus HAROLD, 1871

* lividus (OLIVIER, 1789)

* translucidus PETROVITZ, 1961

Subgenus: Liothorax MoTSCHULSKY, 1860

* kraatzi HAROLD, 1868

Subgenus: Loraphodius REITTER, 1892

* corrugatus PeTROVITZ, 1954

Subgenus: Mecynodes Mulsant et REY, 1870

* kisilkumi SOLSKY, 1876

* stereotypus D. KosHANTSCHIKOV, 1894

Subgenus: Melinopterus MuLSANT, 1842

* consputus CREUTZER, 1799

* edithae REITTER, 1906

* imamae STEBNICKA, 1988

* prodromus (BRAHM, 1790)

* punctatosulcatus hirtipes FISCHER VON WALDHEIM, 1844

Subgenus: Mendidaphodius REITTER, 1901

* lepidulus HaRold, 1866

Subgenus: Mendidius HAROLD, 1868

* beluchistanicus PETROVITZ, 1962

* bidens SOLSKY, 1876 
Subgenus: Mesontoplatys MOTSCHULSKY, 1864

* arabicus HAROLD, 1875

Subgenus: Nialus MuLSANT et REY, 1870

* varians DUFTSCHMIDT, 1805

Subgenus: Nobius MulsanT et REY, 1870

* inclusus REITTER, 1892

Subgenus: Pheaphodius REITTER, 1892

* rectus MotsCHULSKY, 1866

Subgenus: Phalacronotus MOTSCHULSKY, 1860

* flavolimbatus PETROVITZ, 1963

* hilaris HAROLD, 1869

Subgenus: Plagiogonus Mulsant, 1842

* farai BALTHASAR, 1956

* zobeideae Petrovitz, 1980

Subgenus: Planolinus MulsanT et REY, 1870

* fasciatus (OLIVIER, 1789)

Subgenus: Pseuderytus HollandE et THEROND, 1999

* chobauti ClOUËT, 1896

Subgenus: Pseudesymus D’ORBIGNY, 1896

* lucidus KLUG, 1845

Subgenus: Subrinus MulsanT et REY, 1870

* clermonti REITTER, 1907

* sturmi HAROLD, 1870

* vittelinus KLUG, 1845

Subgenus: Trichonotulus BEDEL, 1911

* scrofa (FABRICIUS, 1787)

Subgenus: Volinus MulsanT et REY, 1870

* sticticus (PANZER, 1798)

Genus: Euheptaulacus G. DellaCASA, 1983

* sus (HERBST, 1783)

Genus: Osmanius BRANCO et BARAUD, 1988

* persicus BRANCO et BARAUD, 1988

Genus: Oxyomus DEJEAN, 1833

* sylvestris (SCOPOLI, 1763)

Genus: Trigonoscelus PETROVITZ, 1963

* elbursensis STEBNICKA et GALANTE, 1991

Genus: Turanella SEMENOV, 1905

* latevittis (REITTER, 1887) 
Tribe: Eupariini A. SCHMIDT, 1910

Genus: Ataenius HAROLD, 1867

* horticola HAROLD, 1869

Tribe: Psammodiini MulsanT, 1842

Genus: Granulopsammodius RAKOVIĆ, 1981

* mesopotamicus (PETROVITZ, 1971)

* persicus RAKOVIĆ, 1998

Genus: Leiopsammodius RAKOVIĆ, 1981

* jelineki RAKOVIĆ, 1977

Genus: Pararhyssemus BALTHASAR, 1956

* coluber (MAYET, 1887)

Genus: Pleurophorus MulsAnT, 1842

* anatolicus PETROVITZ, 1961

* apicipennis REITTER, 1892

* arabicus PITTINO et MARIANI, 1986

* pannonicus PETROVITZ, 1961

Genus: Psammodius FALLÉN, 1807

* asper (FABRICIUS, 1775)

* pierottii PITTINO, 1979

Genus: Rhyssemodes REITTER, 1892

* orientalis (MULSANT et GODART, 1875)

* sindicus PitTINo, 1984

Genus: Rhyssemus MULSANT, 1842

* berytensis MARSEUL, 1878

* histrio BALthasar, 1961

* inscitus (WALKER, 1858)

* morgani BÉNARD, 1911

* perissinottoi PITTINO, 1983

* xerxes PITTINO, 1983

Genus: Trichiorhyssemus CLOUËT, 1901

* elegens (PETROVITZ, 1963)

Subfamily: Scarabaeinae LATREILLE, 1802

Tribe: Coprini LEACH, 1815

Genus: Copris GeOFFroy, 1762

Subgenus: Copris GEOFFroY, 1762

* armeniacus FALDERMANN, 1835

* hispanus cavolinii (PETAGNA, 1792)

* lunaris (LinNAEUS, 1758) 
Genus: Heliocopris HoPE, 1837

* midas (FABRICIUS, 1775)

Genus: Metacatharsius MonTREUIL, 1998

* inermis (LAPORTE, 1840)

Genus: Synapsis BATES, 1868

* tmolus (FISCHER VON WALDHEIM, 1821)

Tribe: Gymnopleurini LACORDAIRE, 1856

Genus: Gymnopleurus ILLIGER, 1803

* aciculatsu GEBLER, 1841

* flagellatus (FABRICIUS, 1787)

* geoffroyi mimus BALTHASAR, 1934

* mopsus mopsus (Pallas, 1781)

* mopsus persianus ReITter, 1909

Tribe: Oniticellini KolBE, 1905

Genus: Euoniticellus A. JANSSENS, 1953

* fulvus (GOEZE, 1777)

* pallipes (FABRICIUS, 1781)

Genus: Paroniticellus BALTHASAR, 1963

* festivus (STEVEn, 1809)

Tribe: Onitini LAPORTE DE CASTELNAU, 1840

Genus: Bubas Mulsant, 1842

* bubaloides A. JANSSENS, 1938

Genus: Cheironitis LANDSBERGE, 1875

* granulipennis REITTER, 1909

* haroldi (BALLION, 1870)

* klapperichi (BALTHASAR, 1956)

* moeris (PAllas, 1781)

* pamphilus (MÉNÉTRIES, 1849)

* sterculius (BALLION, 1871)

Genus: Onitis FABRICIUS, 1798

* damoetas Steven, 1806

* humerosus (PALlas, 1771)

Tribe: Onthophagini BURMEISTER, 1846

Genus: Caccobius THOMSON, 1863

Subgenus: Caccobius THOMSON, 1863

* histeroides (MÉNÉTRIES, 1832)

* mundus (MÉNÉTRIES, 1839)

* schreberi (LiNNAEUS, 1767) 
Genus: Digitonthophagus BALTHASAR, 1959

* bonasus (FABRICIUS, 1775)

Genus: Euonthophagus BALTHASAR, 1959

* aeneobrunneus (KABAKOV, 1977)

* amyntas auchenia (REDTENBACHER, 1850)

* amyntas subviolaceus (MÉNÉTRIES, 1832)

* atramentarius (MÉNÉTRIES, 1832)

* conterminus (PETROVITZ, 1971)

* dorbignyi (OLSOUFIEFF, 1900)

* gibbosus (SCRIBA, 1790)

* koshantschikoffi (REITTER, 1891)

* loeffleri (PETROVITZ, 1965)

* mostafatsairi PALESTRINI, VAROLA et Zunino, 1979

* pentaceros (OLSOUFIEFF, 1900)

* pertinax (BALTHASAR, 1959)

* rechingeriorum (MANDL, 1976)

* sulcicollis (REITTER, 1892)

* verberatus ZIANI, 2006

* yama ZIANI, 2006

Genus: Onthophagus LATREILLE, 1802

* hyrcanius KABAKOV, 1982

* liothorax KOSHANTSCHIKOV, 1894

* nitidulus KLUG, 1845

Subgenus: Amphionthophagus MARTIN PIERRA et ZUNINO, 1983

* falzonii GOIDANICH, 1926

Subgenus: Eremonthophagus ZUNINO, 1979

* heydeni HAROLD, 1875

* oberthueri D'ORBIGNY, 1898

* sticticus HAROLD, 1867

* transcaspicus KÖNIG, 1889

Subgenus: Exonthophagus KABAKOV, 2005

* haroldi BALLION, 1871

Subgenus: Furconthophagus ZUNINO, 1979

* variegatus (FABRICIUS, 1798)

Subgenus: Onthophagus LATREILLE, 1802

* illyricus (SCOPOLI, 1763)

* taurus (SCHREBER, 1759) 
Subgenus: Palaeonthophagus ZunINO, 1979

* aerarius ReITTER, 1892

* aleppensis L. REDTENBACHER, 1843

* angorensis PETROVITZ, 1963

* carpanetoi PITtino, 1982

* conspersus ReITtER, 1892

* cruciatus MÉNÉTRIES, 1832

* dorsosignatus D'ORBIGNY, 1898

* excubitor ZIANI et GUDENZI, 2006

* fissicornis STEVEN, 1809

* fissinasus FAIRMAIRE, 1895

* flagrans REITTER, 1892

* fracticornis (PREYSSLER, 1790)

* furciceps MARSEUL, 1869

* gibbulus gibbulus (PALLAS, 1781)

* gibbulus rostrifer REITTER, 1892

* lemur (FABRICIUS, 1781)

* lemuroides D’ORBIGNY, 1898

* liothorax KoshanTSCHIKOV, 1894

* lucidus (ILliger, 1800)

* marginalis marginalis (GEBLER, 1817)

* muelleri P. NovaK, 1921

* nitidulus KLUG, 1845

* osellai PitTino, 1982

* ovatus (LinNAEUS, 1758)

* parmatus ReITTER, 1892

* persianus OlsoufiefF, 1900

* ponticus HAROLD, 1883

* psychopompus ZIANI et MORADI, 2010

* pygargus MOTSCHULSKY, 1845

* rechingeri Petrovitz, 1980

* ruficapillus BRULLÉ, 1832

* sacharovskii OLSOUFIEFF, 1918

* sericatus ReITTER, 1892

* speculifer SOLSKY, 1876

* suturellus BRULLÉ, 1832

* truchmenus iranicus KABAKOV, 2006

* truchmenus truchmenus Kolenati, 1846 
* truchmenus xerxes PetRovitz, 1965

* vacca (LINNAEUS, 1767)

* verticicornis (LAICHARTING, 1781)

* viridis MÉNÉTRIES, 1832

* viriditinctus REITTER, 1892

* vlasovi MEDVEDEV, 1958

Subgenus: Relictonthophagus KABAKOV, 1979

* zagrosicus KABAKOV, 2006

Tribe: Scarabaeini LATREILLE, 1802

Genus: Scarabaeus LINNAEUS, 1758

Subgenus: Ateuchetus BEDEL, 1892

* armeniacus MÉNÉTRIES, 1832

Subgenus: Scarabaeus LINNAEUS, 1758

* acuticollis (MOTSCHULSKY, 1849)

* andrewesi (FELSCHE, 1907)

* babori BALTHASAR, 1934

* bannuensis A. JANSSENS, 1940

* brahminus (LAPORTE DE CASTELNAU, 1840)

* carinatus (GEBLER, 1841)

* cristatus cristatus FABRICIUS, 1775

* cristatus villiersi BARAUD, 1973

* pius (ILLIGER, 1803)

* sacer LINNAEUS, 1758

* transcaspicus STOLFA, 1938

* typhon FISCHER VON WALDHEIM, 1823

* wilsoni WATERHOUSE, 1890

* winkleri StOLFA, 1938

Tribe: Sisyphini MulsanT, 1842

Genus: Sisyphus LATREILLE, 1807

Subgenus: Sisyphus LATREILLE, 1807

* schaefferi schaefferi (LINNAEUS, 1758)

Subfamily: Euchirinae BURMEISTER, 1842

Genus: Propomacrus NEWMAN, 1837

* bimucronatus (PALlAS, 1781) 
Subfamily: Melolonthinae SAMOUELLE, 1819

Tribe: Diplotaxini KIRBY, 1837

Genus: Apogonia KIRBY, 1819

* belutschistanica PeTrovitz, 1958

Tribe: Hopliini LATREILLE, 1829

Genus: Hoplia ILLIGER, 1803

Subgenus: Decamera MulsANT, 1842

* jakobsoni REITTER, 1903

Subgenus: Hoplia IlLIGER, 1803

* caucasica KolENATI, 1846

* hyrcana MEDVEDEV, 1952

Tribe: Melolonthini SAMOUELLE, 1819

Genus: Cryptotrogus KRAATZ, 1888

* miksici Petrovitz, 1965

? niveus (HAMPE in WAGNER, 1852)

* orita (REITTER, 1902)

* zarudnianus (SEMENOV et MEDVEDEV, 1936)

Genus: Cyphonotus FISCHER VON WALDHEIM, 1824

* testaceus (PALlAS, 1781)

Genus: Cyphonoxia REITTER, 1889

* glasunowi SEMENOV, 1897

* kermanensis SEMENOV et MEDVEDEV, 1936

* praestabilis REITTER, 1889

* tatianae SEMENOV et MEDVEDEV, 1936

? weisei (KRAATZ, 1888)

* zarudnyi SEMENOV et MEDVEDEV, 1936

Genus: Medeotrogus KEITH, 2001

* linnavuorii KeITH, 2001

Genus: Melolontha FABRICIUS, 1775

* kraatzi REITTER, 1906

* macrophylla FISCHER VON WALDHEIM, 1830

Genus: Microphylla KRAATZ, 1890

* paupera (HAMPE, 1852)

Genus: Polyphylla HARRIS, 1841

Subgenus: Polyphylla HARRIS, 1841

* olivieri LAPORTE, 1840

* persica BRENSKE, 1902

Subgenus: Xerasiobia MEDVEDEV, 1951

* adspersa MOTSCHULSKY, 1854 
Tribe: Pachydemini BuRMEISTER, 1855

Genus: Alaia Petrovitz, 1980

* sexdentata Petrovitz, 1980

Genus: Asiactenius NiKOLAJEV, 2000

* tekkensis (REITTER, 1889)

* tokgajevi (MEDVEDEV, 1962)

Genus: Atanyproctus PETROVITZ, 1954

* ernae Petrovitz, 1980

* opacipennis Petrovitz, 1968

* simplicitarsis PETROVITZ, 1954

Genus: Falsotoclinius KeITH et MONTREUIL, 2004

* fragilis (PETROVITZ, 1980)

* richteri (PETROVITZ, 1958)

* schauffelei (PETROVITZ, 1958)

Genus: Otoclinius BRENSKE, 1897

* gracilipes BRENSKE, 1897

Genus: Phalangonyx REITTER, 1889

* coniceps REITTER, 1889

* hormoziaensis (PETROVITZ, 1980)

* mirzayani (Petrovitz, 1968)

* semenovi (MEDVEDEV, 1952)

Genus: Tanyproctoides PETROVITZ, 1971

Subgenus: Odontiellus KeITH et MONTREUIL, 2004

* mirzayansinus KeITH et MonTREuIL, 2004

Genus: Tanyproctus MÉNÉTRIES, 1832

* beludschistanus PETROVITZ, 1968

? carbonarius FALDERMANN, 1835

* confinis MOTSCHULSKY, 1860

* eghtedari Petrovitz, 1980

* ganglbaueri (BRENSKE, 1897)

* inflatus Motschulsky, 1860

* iranicus Petrovitz, 1968

* kermanshahensis PeTROVITZ, 1980

* persicola ReITTER, 1909

* persicus (MÉNÉTRIES, 1832)

* rufidens (MARSEUL, 1879)

* satanas REITTER, 1902

* varians PETROVITZ, 1980

* zartoshti PetRovitz, 1980 
Tribe: Rhizotrogini BURMEISTER, 1855

Genus: Amphimallon LATREILLE, 1825

* beludschistanus BRENSKE, 1897

* leuthneri ReITTER, 1902

* solstitiale setosum (REITTER, 1902)

* vernale persicum PeTROVITZ, 1970

Genus: Aplidia HoPE, 1837

* iranica (Petrovitz, 1970)

* tarsensis KRAATZ, 1882

Genus: Chilotrogus REITTER, 1905

* farsensis KeITH, 2003

* panotrogoides ReITTER, 1905

* phasmellus BIGGS, 1945

Genus: Chioneosoma KraAtZ, 1891

* deserti BARAUD, 1987

? porosum (FISCHER VON WALDHEIM,1824)

* vulpinum (GYLLENHALL, 1817)

Genus: Holochelus ReITTER, 1889

Subgenus: Holochelus REITTER, 1889

* fallaciosus (NONVEILLER, 1965)

* fallax fallax (MARSEUL, 1879)

* nadaii KeITH, 2003

* pseudonadaii KeITH, 2003

Subgenus: Miltotrogus REITTER, 1902

* fusculus (NONVEILLER, 1965)

* kerimi (REY, 1994)

* parvus (NONVEILLER, 1965)

Genus: Lasiexis SEMENov et Medvedev, 1936

* dilaticollis (BALLION, 1870)

* glabrithoracica KEITH, 2003

* persicola Petrovitz, 1980

* primaeveris Semenov et Medvedev, 1936

Genus: Madotrogus ReITTER, 1902

Subgenus: Dasytrogus REITTER, 1902

* glabricollis (REITTER, 1888)

Subgenus: Madotrogus REITTER, 1902

* bicolor Petrovitz, 1970 
Genus: Panotrogus REITTER, 1902

? myschenkovi (BALLION, 1871)

* petrovitzi BARAUD, 1989

Genus: Rhizotrogus LATREILLE, 1825

? aestivus (OLIVIER, 1789)

* torrulosus WALTL, 1838

Genus: Xanthotrogus REITTER, 1902

* cariniclypeatus KEITH, 2003

* leonhardi ReITTER, 1909

* peratratus (REITTER, 1909)

* semnanensis $\mathrm{KEITH,} 2003$

* sieversi (REITTER, 1896)

* tanyproctoides (REITTER, 1906)

Subfamily: Sericinae KIRBY, 1837

Genus: Maladera MulsANT et REY, 1871

Subgenus: Cephaloserica BRENSKE, 1900

* insanabilis (BRENSKE, 1894)

* nagporeana (BRENSKE, 1898)

Subgenus: Eusericula REITTER, 1902

* ahrensi KEITH, 2005

* farsensis PETROVITZ, 1980

* girardi KEITH et AHRENS, 2002

* hodkovae NiKODÝM et KRÁL, 1998

* jiraskovae SENHAL, 2008

Subgenus: Macroserica MEDVEDEV, 1952

* armeniaca REITTER, 1896

* beluchestanica Petrovitz, 1971

* punctatissima (FALDERMANN, 1835)

Genus: Omaloplia STEPHENS, 1830

Subgenus: Acarina BARAUD, 1965

* labrata (BURMEISTER, 1855) 
Subfamily: Rutelinae MACLEAY, 1819

Tribe: Adoretini BURMEISTER, 1844

Genus: Adoretus LAPORTE, 1840

Subgenus: Adoretus LAPORTE, 1840

* abdolrezagharibi PETROVITZ, 1980

* iranicus Petrovitz, 1958

* nigrifrons (STEVEN, 1809)

* persicus REITTER, 1889

* petrovitzi BARAUD, 1975

* scabiosus Petrovitz, 1980

* sistanicus PETROVITZ, 1970

Subgenus: Lepadoretus REITTER, 1903

* kashmarensis KEITH, 2001

* vastus PETROVITZ, 1958

Genus: Clipadoretus OHAUs, 1941

* dentatus Petrovitz, 1967

* persicus OHAUS, 1941

Genus: Lissadoretus ARROw, 1917

* pallidus ARrow, 1917

Genus: Phaeadoretus REITTER, 1903

* comptus (MÉNÉTRIES, 1849)

* iranicus MACHATSCHKE, 1960

* schirasensis PETROVITZ, 1970

* syriacus (BLANCHARD, 1851)

Tribe: Anomalini MulsANT, 1842

Genus: Anisoplia FISCHER VON WALDHEIM, 1824

Subgenus: Anisoplia FISCHER VON WALDHEIM, 1824

* agnata REITTER, 1889

* faldermanni REITTER, 1883

* farraria antoniae REITTER, 1889

* persiaca BICZÓK, 1940

* reitteriana SEMENOV, 1903

* signata signata FALDERMANN, 1835

* turcomana ZAITZEV, 1917

Subgenus: Autanisoplia MEDVEDEV, 1949

* austriaca major REITTER, 1889

* austriaca persica PETROVITZ, 1980 
Subgenus: Pilleriana BARAUd, 1991

* campicola MÉNÉTRIES, 1832

Genus: Anomala SAMOUELlE, 1819

* splendida MÉNÉTRIES, 1832

Genus Asiopertha MACHATSCHKE, 1957

* ganglbauberi (REITTER, 1885)

* nazarena (MARSEUL, 1878)

Genus: Blitopertha REITTER, 1903

* nigripennis (REITTER, 1888)

Genus: Brancoplia BARAUD, 1986

* leucaspis leucaspis (LAPORTE DE CASTELNAU, 1840)

Genus: Chaetopteroplia MEDVEDEV, 1949

* syriaca BURMEISTER, 1844

Genus: Pharaonus Blanchard, 1851

* varicoloreus (BURMEISTER, 1844)

Subfamily: Dynastinae MACLEAY, 1819

Tribe: Oryctini MuLSANT, 1842

Genus: Oryctes ILLIGER, 1798

Subgenus: Eremoryctes SEMENOV et MEDVEDEV, 1932

* ata SEMENOV et MEDVEDEV, 1932

Subgenus: Oryctes ILLIGER, 1798

* nasicornis hindenburgi MINCK, 1915

* nasicornis illigeri MINCK, 1915

* nasicornis latipennis MOTSCHULSKY, 1845

* nasicornis shiraticus ENDRÖDI et PETROVITZ, 1974

* nasicornis transcaspicus ENDRÖDI, 1938

Subgenus: Rykanoryctes MINCK, 1919

* agamemnon matthiesseni REITTER, 1907

* elegans PRELL, 1914

* richteri PeTROVITZ, 1958

Tribe: Pentodontini MulsanT, 1842

Genus: Pentodon HoPe, 1837

* algerinus bispinifrons REITTER, 1894

* algerinus dispar BAUDI, 1870

* bidens sulcifrons KÜSTER, 1848

* caminarius caminarius (FALDERMANN, 1835)

* caminarius elatus KÜSTER, 1847 
* idiota idiota (HERBST, 1789)

* minutus ReITTER, 1887

* quadridens distantidens ReITTER, 1899

Genus: Phyllognathus EsCHSCHOLTZ, 1830

* excavatus (FORSTER, 1771)

Genus: Podalgus BURMEISTER, 1847

* cuniculus infantulus SEMENOV, 1889

Subfamily: Cetoniinae LEACH, 1815

Tribe: Cetoniini LEACH, 1815

Genus: Aethiessa BURMEISTER, 1842

* inhumata (Gory et Percheron, 1833)

* mesopotamica BURMEISTER, 1842

* zarudnyi KISERITZKY, 1910

Genus: Cetonia FABRICIUS, 1775

Subgenus: Cetonia FABRICIUS, 1775

* aeratula ReITTER, 1891

* aurata aurata (LinNAEUs, 1761)

* aurata pallida (DRURY, 1773)

* sexguttata OLSOUFIEFF, 1916

Genus: Heterocnemis AlBERS, 1853

Subgenus: Celidotella REITTER, 1909

* matthiesseni (REITTER, 1909)

Genus: Oxythyrea MuLSANT, 1842

* cinctella cinctella (SCHAUM, 1841)

* cinctella taftanensis MONTREUIL et LEGRAND, 2008

* funesta (PODA, 1761)

Genus: Protaetia BURMEISTER, 1842

Subgenus: Cetonischema REITTER, 1898

* speciosa cyanochlora (SCHAUER, 1841)

* speciosa jousselini (Gory et PerCheron, 1833)

* speciosa speciosa (ADAMS, 1817)

Subgenus: Eupotosia MIKŠIĆ, 1954

* affinis affinis (ANDERSCH, 1797)

* affinis pseudospeciosa (MEDVEDEV, 1964)

* affinis pyrodera (REITTER, 1891) 
Subgenus: Netocia CosTA, 1852

* aethiessina (REITTER, 1891)

* araratica (REITTER, 1891)

* asiatica (FALDERMANN, 1835)

* golestanica (ALEXIS et DELPONT, 2001)

* jakli (MONTREUIL et LEGRAND, 2008) comb. nov.

* karbalayei (ALEXIS et DELPONT, 2001)

* khorasanica (MONTREUIL et LEGRAND, 2008) comb. nov.

* schelkovnikovi (ZAITZEV, 1918)

* subpilosa (DESBROCHERS, 1869)

* trojana godeti (GORY et PERCHERON, 1833)

* ungarica armeniaca (MÉNÉTRIES, 1832)

* ungarica auliensis (REITTER, 1901)

* ungarica ignisternum (REITTER, 1891)

* ungarica persica (KRAATZ, 1886)

* vidua (GORY et PERCHERON, 1833)

* zagrosica (AlEXIS et DELPONT, 2001)

Subgenus: Potosia Mulsant et REY, 1971

* cuprea caucasica (KolENATI, 1846)

* cuprea mehrabii (MONTREUIL et LEGRAND, 2008) comb. nov.

* flutschiana (MONTREUIL et LEGRAND, 2008) comb. nov.

* funebris funebris (Gory et PERCHERON, 1833)

* hieroglyphica depressiuscula (REITTER, 1891)

* hieroglyphica hieroglyphica (MÉNÉTRIES, 1832)

* keithi (MONTREUIL et LEGRAND, 2008) comb. nov.

* splendidula diademata (REITTER, 1898)

* splendidula kermanensis (MONTREUIL et LEGRAND, 2008) comb. nov.

* splendidula splendidula (FALDERMANN, 1835)

Genus: Stalagmosoma BURMEISTER, 1842

* albellum (PALLAS, 1781)

Genus: Tropinota MULSANT, 1842

Subgenus: Epicometis BURMEISTER, 1842

* hirta suturalis REITTER, 1913

* senicula (MÉNÉTRIES, 1832)

* spinifrons REITTER, 1889

* turanica REITTER, 1889

* villiersis BARAUD, 1984 


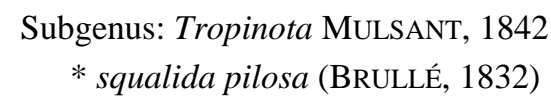

Tribe: Trichini FLEMING, 1821

Genus: Gnorimus LEPELETIER et SERVILLE, 1828

* subcostatus (MÉNÉTRIES, 1832)

Genus: Trichius FABRICIUS, 1775

* abdominalis MÉNÉTRIES, 1832

Tribe: Valgini MULSANT, 1842

Genus: Valgus SCRIBA, 1790

* hemipterus (LINNAEUS, 1758)

\section{REFERENCES}

BARARI H. 2001. Insects of Iran. The list of Coleoptera in the Insect Collection of Plant Pests \& Diseases Research Institute. Coleoptera (XXIV): Scarabaeoidea. Group: Laparosticti. Fam.: 39, 40, 44, 46 (Geotrupidae, Hybosoridae, Orphnidae, Scarabaeidae and Aphodiidae). Plant Pests \& Diseases Research Institute Tehran 7: 1-64.

Barari H., Kharrazi PaKdel A., Boroumand H. 1998a. A faunistic survey of Laparosticti group (Col.: Scarabaeidae) in Karaj area. [in:] Proceedings of $13^{\text {th }}$ Iranian Plant Protection Congress, 23-27 August, Karadj, Iran. Junir Agriculture College of Karadj, Karadj, 202.

Barari H., Kharrazi Pakdel A., Boroumand H. 1998b. A faunistic survey of Pleurosticti group (Col.: Scarabaeidae) in Karaj area. [in:] Proceedings of $13^{\text {th }}$ Iranian Plant Protection Congress, 23-27 August, Karadj, Iran. Junir Agriculture College of Karadj, Karadj, 203.

BARAUD J. 1968. Contribution à la faune de l'Iran. 10. Coleoptera: Scarabaeoidea. Annales de la Société Entomologique de France (N.S.) 4(4): 915-925.

BORTESI O., ZUNINO M. 1974. Les résultats de l'expédition entomologique tchéchoslovaque-iranienne l'Iran en 1970. No. 10: Les Onthophagus du sous-genre Euonthophagus BALTH. (Coleoptera, Scarabaeoidea). Acta Entomologica Musei Nationalis Pragae Suppl. 6: 105-107.

Ghahari H., Bunalski M., TABARi M., Ostovan H. 2011. Scarab beetles (Coleoptera: Scarabaeidae) collected in Iranian rice fields and surrounding grasslands. Polish Journal of Entomology, 80(4): 465-474.

Hassan Zadeh B., ZehZad B., Farhang B., Majnounian H., Goshtasb H. 1993. Golestan National Park. Department of Environment Publication, Fardin.

KABAKOv O.N. 1982. Contribution to the fauna of lamellicorn beetles of the genus Onthophagus from Middle Asia, Afghanistan and Iran. Trudy Zoologicheskogo Instituta Akademii Nauk SSSR 110(1): 8-19. (in Russian)

KeIth D., Ahrens, D. 2002. Zur Kenntnis der Arten der Gattung Maladera Mulsant et Rey, 1871, aus dem Iran (Coleoptera: Scarabaeidae, Sericinae). Entomologische Zeitschrift 112(7): 194-197. 
Keith D., Uliana, M. 2008. Contribution to the knowledge of Iranian Pygopleurus Motschulsky, with description of $P$. rapuzzii n. sp. (Coleoptera: Scarabaeoidea: Glaphyridae). Zootaxa 1825: 59-64.

LöBl I., Smetana A. (eds) 2006. Catalogue of Palaearctic Coleoptera. Volume 3. Scarabaeoidea Scirtoidea - Dascilloidea - Buprestoidea - Byrrhoidea. Apollo Books, Stenstrup.

Modarres Awal M. 1997. Family Scarabaeidae. [in:] Modarres Awal M. (ed.). List of agricultural pests and their natural enemies in Iran. Ferdowsi University Press, Mashhad, $186-192$.

ModARres Awal M. 2006. Preliminary studies on Scarabaeoidea (Coleoptera) fauna of Razavi Khorasan province of Iran. Türkiye Entomoloji Dergisi 30(3): 163-172.

Montreuil O., SerRi S. 2007. Description of a new Pygopleurus species (Col.: Glaphyridae), with a list of recorded species of the genera Pygopleurus and Eulasia from Iran. Journal of Entomological Society of Iran 26(2): 1-6.

Moradi GHARAKHLOO M., Ziani S. 2009. Occurrence of scarab beetles inside rodent burrows in some parts of Iran (Coleoptera: Scarabaeidae). Zoology in the Middle East 46(1): 95-98.

MORADI GHARAKHLOO M., ZIANI S. 2010. Iranian dung beetles (Coleoptera: Scarabaeidae) associated with rodent burrows: list of collected species and some additional comments. Boletín de la Sociedad Entomológica Aragonesa 47: 165-170.

Mowlavi G., Mikaeili E., Mobedi I., Kia E.B., Masoomi L., Vatandoost H. 2008. Scarab beetle (Coleoptera: Scarabaeidae) fauna in Ardabil province, north west Iran. Iranian Journal of Arthropod-Borne Disease 2(2): 35-41.

NiKODÝM M., KRÁL D. 1998. Results of the Czech biological expedition to Iran. Part 3. Coleoptera: Glaphyridae and Scarabaeidae: Sericinae. Acta Societatis Zoologicae Bohemoslovacae 62(4): 343-351.

NiKOLAYEV G.V. 1987. Scarabaeidae (Coleoptera) of Kazakhstan and Middle Asia. Izdatel'stvo "Nauka" Kazakhskoy SSR, Alma-Ata. (in Russian)

Petrovitz R. 1954. Ergebnisse der Österreichischen Iran-Expedition 1949/50. Coleoptera, IV. Teil. Scarabaeidae. Sitzungsberichte der Österreichischen Akademie der Wissenschaften 163: 261275.

Petrovitz R. 1958. Beitrag zur Kenntnis der Scarabaeiden-Fauna des Iran. Stuttgarter Beiträge zur Naturkunde 8: 1-12.

Petrovitz R. 1980. Österreichische entomologische Expeditionen nach Persien und Afghanistan. Beiträge zur Coleopterenfauna. Teil XII. Weiteres über Lamellicornia aus Iran. Annalen des Naturhistorischen Museum in Wien 83: 597-638.

RATCLIFFE B.C., PAUlSEN M.J. 2008. The Scarabaeoid beetles of Nebraska. Bulletin of the University of Nebraska State Museum 22: 1-569.

RAtCliffe B.C., Jameson M.L., Smith A.B.T. 2002. Scarabaeidae LATReille, 1802. [In:] ARNETt R.H., Thomas M.C., Skelley P.E., Frank J.H. (eds). American Beetles. Vol. 2. CRC Press, Boca Raton, 39-42. 
WoodrufF R.E. 1973. The Scarab beetles of Florida (Coleoptera: Scarabaeidae). Part 1. The Laparosticti (Subfamilies: Scarabaeinae, Aphodiinae, Hybosorinae, Ochodaeinae, Geotrupinae, Acanthocerinae). Florida Department of Agriculture and Consumer Services Contribution No. 260: $1-220$.

Yarmand H., Mehrabi A., Montreuil O. 2012. Faunistic survey of the some scarab beetles in Ghazvin province. [in:] Proceedings of $20^{\text {th }}$ Iranian Plant Protection Congress, 4 Sep. -7 Sep. 2012, Shiraz, Iran, 129.

ZAIRI M. 1976. List of Scarabaeidae from province of Fars. Part 4. Journal of Entomological Society of $\operatorname{Iran} 3(1-2)$ : $125-127$.

ZIANI S. 2006. Remarks on some near eastern Euonthophagus species with the description of two new species from Iran (Insecta Coleoptera Scarabaeidae: Onthophagini). Quaderno di Studi e Notizie di Storia Naturale della Romagna 23: 95-130.

ZIANI S. 2011. A new Iranian Onthophagus species belonging to the O. semicornis group (Coleoptera: Scarabaeidae: Onthophagini). Zootaxa 2907: 47-50.

ZiANi S., GUdENZI I. 2000. About some Onthophagus LATREILLE, 1802 (sensu lato) collected during a trip to Iran (Insecta, Coleoptera, Scarabaeidae). Quaderno di Studi e Notizie di Storia Naturale della Romagna 13(Suppl.): 17-26.

ZiAni S., GudenZI I. 2006. Studies on palearctic Onthophagus associated with burrows of small mammals. I. O. furciceps, O. kindermanni, O. vitulus and closely related species (Coleoptera Scarabaeidae). Bollettino della Società Entomologica Italiana 138(3): 207-248.

ZiAni S., Gudenzi I. 2007. Studies on Palearctic Onthophagus associated with burrows of small mammals. II. O. semicornis and closely related species (Coleoptera: Scarabaeidae: Onthophaginae). Folia Heyrovskyana Ser. A 15(2): 89-114.

ZIANI S., GudenZI I. 2009. Studies on Palearctic Onthophagus associated with burrows of small mammals. III. O. aerarius and closely related species (Coleoptera Scarabaeidae). Bollettino della Società Entomologica Italiana 141(1): 29-44.

Ziani S., Moradi GHarakhloo M. 2010. Studies on Palearctic Onthophagus associated with burrows of small mammals. IV. A new Iranian species belonging to the furciceps group (Coleoptera, Scarabaeidae, Onthophagini). ZooKeys 34: 33-40.

Received: 1 February 2014

Accepted: 6 April 2014 